\title{
StaphMap Tyrol - pre-study on the prevalence of methicillin-resistant Staphylococci (MRSA) in Tyrol
}

\section{Oberhauser Heidi ${ }^{1}$}

fhg - Health University of Applied Sciences Tyrol

Innrain 98, 6020 Innsbruck, Austria

heidi.oberhauserefhg-tirol.ac.at

\section{Lutz-Nicoladoni Christina}

fhg - Health University of Applied Sciences Tyrol

Innrain 98, 6020 Innsbruck, Austria

christina.lutz-nicoladoniefhg-tirol.ac.at

\begin{abstract}
Staphylococci are gram-positive bacteria, physiologically residing on human and animal skin and mucosa. Most clinically relevant is Staphylococcus aureus (S. aureus), a facultative pathogenic organism provoking infections of skin and wounds, but also severe systemic infections in immunosuppressed patients. Methicillin-resistant strains of S. aureus (MRSA), associated with the health care system, termed hospital acquired (HA)-MRSA, have been a major hospital hygiene problem for decades and are the major cause of nosocomial infections. In a population without any contact to the health care system community acquired (CA)-MRSA, mostly higher pathogenic has been detected more frequently during the last years. Additionally, livestock-associated (LA)MRSA, associated with massive use of antibiotics in industrial livestock farming, is found in man and beast.
\end{abstract}

With this pre-study to the project StaphMap Tyrol we investigated the prevalence of methicillinsensitive S. aureus (MSSA) and MRSA in healthy probands. Pupils and teachers of seven Tyrolean high schools $(n=173)$ from different districts took nasal swabs of themselves, which were analysed during a teaching project in the laboratories of the fhg and at the Section of Hygiene and Medical Microbiology. A further objective was to enable pupils to take part in a scientific project and to learn basic microbiological techniques. First preliminary data, obtained from this sampling reveal a prevalence of $21,4 \% \mathrm{~S}$. aureus and 1,2\% MRSA. Further investigations based on a representative sample size as well as genetic and epidemiological analysis will follow.

5th Austrian Citizen Science Conference 2019 (ACSC2019)

26-28, June, 2019

Obergurgl, Austria

\section{${ }^{1}$ Speaker}




\section{Introduction}

\subsection{Background}

Staphylococci are widespread gram-positive bacteria physiologically colonising human and animal skin and mucosa. $S$. aureus is the major human facultative pathogen causing skin- and wound-infections but also severe systemic infections in immunosuppressed patients and is the main cause for nosocomial infections [1]. In asymptomatic humans, the bacteria can be isolated from different anatomical niches but lives primarily in the nostrils. One reason for the high prevalence of $S$. aureus is the increasing development of resistance to antibiotics, circumventing effective treatment of infections [2]. Throughout the world, prevalence of methicillin-resistant $S$. aureus (MRSA) has rapidly increased during the last decades [3]. The resistance is due to expression of altered penicillin-binding proteins with reduced affinity to antibiotics, namely penicillin-binding protein $2 \mathrm{a}(\mathrm{PBP} 2 \mathrm{a})$ and $\mathrm{PBP} 2 \mathrm{c}$, encoded by the genes mec $\mathrm{A}$ or. mec $\mathrm{C}$ respectively [3].

Hospital acquired (HA)-MRSA infections are defined as those that have onset in the hospital environment and long-term-care facilities. In addition, MRSA infections increasingly occurred in humans with no contact to the health care system and are called community acquired (CA)-MRSA [4]. CA-MRSA is commonly expressing the toxin panton-valentine leukocidin (PVL), rendering higher pathogenicity as HA-MRSA and causing severe infections even in not-immunosuppressed people [5]. Other community-dwelling MRSA infections have been observed in the context of industrial farming. Those so-called livestock-associated (LA)-MRSA exhibit similar pathogenicity as HA-MRSA. The three types can be differentiated by their structural and functional genomic traits [6].

Whereas HA-MRSA is well investigated, little is known about the prevalence and distribution of CA-MRSA/LA-MRSA within the healthy population. One study including data from eight European countries revealed a prevalence for MRSA of 1,5\% for non-hospitalized Austrians [4].

\subsection{Aim of the study}

Main objective of the study is to levy the prevalence of methicillin-sensitive and methicillinresistant S. aureus (MSSA/MRSA) in the healthy Tyrolean population.

Specific aim 1 is the elicitation of the differences in MRSA-prevalence due to different geographical regions, age, contact to livestock/domestic animals and contact to the health care sector.

Specific aim 2 is the involvement of citizens in the project by collecting the probes (nasal swabs). In this pre-study, high-school pupils collected the samples and performed primary steps of the microbiological analysis.

\section{Material and Methods}

The project was planned relating to the descriptive framework of citizen science projects due to Lea Den Broeder et al., where aim of citizen engagement, level of participation and (geographical) size are the main characteristics. [7] Within a teaching project, seven school classes of almost all Tyrolean districts voluntarily participated. First step was to visit pupils and teachers 
at their school to inform them about the aim of the project, sample collection, microbiological and scientific methods by giving a talk and powerpoint presentation. Subsequently, pupils and teachers $(\mathrm{n}=173)$ were invited to a lab day at the fhg (department Biomedical Sciences), where they took nasal swabs of themselves after demonstration and under instruction of Biomedical Scientists and filled in a questionnaire (age, sex, residence, exposure-relevant information like animal contact and/or contact to healthcare systems). For ethical reasons guardians of minor pupils signed the declaration of consent for taking the non-invasive nasal swabs. For ensuring privacy, nasal swabs and questionnaire were consecutively numbered and assignment to a single person is deposited solely at the medical project manager, so that for a potential positive result (MRSA) the pupil could be contacted and therapy induced.

The pupils inoculated their own swab to different culture media for further investigation: columbia broth for enrichment culturing, mannitol-salt-agar for detecting S. aureus, Brilliance MRSA-2-agar for differentiating MSSA and MRSA. All cultures were sent to the project partner at the Section for Hygiene and Medical Microbiology (Innsbruck) to identify MSSA and MRSA. $S$. aureus colonies were verificated with MALDI BiotyperTM. Susceptibility testing was performed applying disk dilution tests according to EUCAST guidelines and on VITEK ${ }^{\circledR}$ Compact.

The pupils were instructed in different microbiological techniques with prepared (notinfectious) samples. They learned how to differentiate bacteria by growth, appearance, biochemical reactions (catalase, oxidase) and learned how to interpret an antibiogram. All these demonstrations and practical exercises were supported with handouts.

\section{Results}

The topic of MRSA is of widespread interest not only for hospital and health care but also for citizens. A consequent implementation of adequate infection control supports the confining of MRSA infections, as demonstrated in the Netherlands and Scandinavian countries [6]. Probands benefit from participation since potentially detected MRSA colonisation can easily be treated by applying an antibiotic nasal ointment for one week [8].

Teaching project at the fhg-laboratories: Seven Tyrolean high-school classes collaborated as part of their biology lessons in the project StaphMap Tirol. The project staff informed the pupils about MRSA, instructed them in microbiological methods as well as scientific procedures, in particular specimen collection. Pupils showed interest in the topic and were skilful in laboratory work. Pupils had to document all techniques in a project report for the biology lessons. The authors sent the interim report to all teachers for discussing the results with the pupils.

MSSA/MRSA prevalence: Preliminary data obtained from the small number of samples $(n=173)$ within this pre-study revealed a prevalence of $S$. aureus colonisation of $21,4 \%$ and MRSA prevalence of $1,2 \%$. These numbers are in accordance with data regarding healthy populations from other European countries [4].

\section{Further investigation}

Due to a representative and stratified sample size of estimated 3000 probes, nursing-students of the fhg will collect nasal swabs of adults in all Tyrolean districts.

The detection of MSSA and MRSA will be performed as described before, the molecular typing of all detected MRSA strains will be examined for the presence of resistant-genes. 
Applying pulsed-field gel electrophoresis allows classifying the MRSA strains epidemiologically, so that the geographical map can be drawn. Application of ethical approval for the project is already granted.

\section{Acknowledgements}

We thank Anna Sprenger for the initiate idea and preliminary concept, the pupils and teachers for their enthusiasm and collaboration and the staff of the Section for Hygiene and Medical Microbiology, in particular Dr. Dorothea Orth-Höller and Verena Fleischer for analysing the probes.

This study is funded by a grant of the Tyrolean Science Fund (GZ: UNI-0404/2341).

\section{References}

[1] C.E. Muenks et al., Diversity of Staphylococcus aureus strains colonizing various niches of the human body, J Infect 72 (2016) 006 doi: 10.1016/j.jinf.2016.03.015

[2] M. Monaco et al., Worldwide epidemiology and antibiotic resistance of Staphylococcus aureus, Curr Top Microbiol Immunol 409 (2017) doi: 10.1007/82_2016_3

[3] N.A. Turner et al., Methicillin-resistant Staphylococcus aureus: an overview of basic and clinical research, Nat Rev Microbiol 17 (2019) 004 doi: 10.1038/s41579-018-0147-4

[4] E. Bijnen van et al., Antibiotic Exposure and Other Risk Factors for Antimicrobial Resistance in Nasal Commensal Staphylococcus aureus: An Ecological Study in 8 European Countries, PLoS One 10 (2015) 008 doi: 10.1371/journal.pone.0135094

[5] F. Vandenesch et al., Community-acquired Methicillin-Resistant Staphylococcus aureus Carrying Panton-Valentine Leukocidin Genes: Worldwide Emergence, Emerg Infect Dis 9 (2003) 008 doi: 10.3201/eid0908.030089

[6] C. Cuny et al., Livestock-Associated MRSA: the Impact on Humans, Antibiotics 4 (2015) doi: 10.3390/antibiotics4040521

[7] L. Broeder Den et al., Citizen Science for public health, Health Promotion International 33 (2018) doi: 10.1093/heapro/daw086

[8] G. Pichler et al., MRSA prevalence rates detected in a tertiary care hospital in Austria and successful treatment of MRSA positive patients applying a decontamination regime with octenidine, Eur J Clin Microbiol Infect Dis 37 (2018) doi: 10.1007/s10096-017-3095-4 